\title{
Lipschitz continuity of solutions of Poisson equations in metric measure spaces
}

\author{
Renjin Jiang
}

\begin{abstract}
Let $(X, d)$ be a pathwise connected metric space equipped with an Ahlfors $Q$-regular measure $\mu, Q \in[1, \infty)$. Suppose that $(X, d, \mu)$ supports a 2-Poincaré inequality and a SobolevPoincaré type inequality for the corresponding "Gaussian measure". The author uses the heat equation to study the Lipschitz regularity of solutions of the Poisson equation $\Delta u=f$, where $f \in L_{\mathrm{loc}}^{p}$. When $p>Q$, the local Lipschitz continuity of $u$ is established.
\end{abstract}

\section{Introduction}

Let $(X, d)$ be a pathwise connected, proper metric measure space, where proper means: each closed ball in $X$ is compact. Given a domain $\Omega \subseteq X$ and $u$ a measurable function on $\Omega$, a nonnegative Borel function $g$ is called an upper gradient of $u$ on $\Omega$, if

$$
|u(x)-u(y)| \leq \int_{\gamma} g d s
$$

for all $x, y \in \Omega$ and each rectifiable curve $\gamma:[0, l] \rightarrow \Omega$ that joins $x$ and $y$. Further, a metric measure space $(X, d, \mu)$ is said to support a (weak) $p$-Poincaré inequality, if there exist $C_{P}>0$ and $\lambda \geq 1$ such that for every ball $B(x, r) \subseteq X$ and for each continuous function $u$ and every upper gradient $g$ of $u$ on $B(x, \lambda r)$,

$$
f_{B(x, r)}\left|u(y)-u_{B}\right| d \mu(y) \leq C_{P} r\left(f_{B(x, \lambda r)} g(y)^{p} d \mu(y)\right)^{1 / p},
$$

where and in what follows, for each ball $B \subset X, u_{B}=f_{B} u d \mu=\mu(B)^{-1} \int_{B} u d \mu$; see [13] for details.

By using the upper gradient, Shanmugalingam [18] introduced the first-order Sobolev spaces on $X$, i.e., the Newtonian (Sobolev) space $N^{1, p}(X, \mu)$. For convenience, we denote the local Newtonian spaces and Newtonian spaces with zero boundary values by $N_{\text {loc }}^{1, p}$ and $N_{0}^{1, p}$, respectively (see Section 2 for details). We note that it was proved in [18] that the Newtonian (Sobolev) spaces $N^{1, p}(X, \mu)$ coincide with the Sobolev spaces introduced by Cheeger [6] for $p>1$. From [6], for each $u \in N^{1, p}(X, \mu)$, we can assign a differential $D u$, which is called Cheeger derivative of $u$ following [15]; see Subsection 2.1 below. Notice that for Lipschitz functions $u$, the inner product

2000 Mathematics Subject Classification. 31C25; 31B05; 35B05; 35B45

Key words and phrases. Lipschitz regularity; Poincaré inequality; Newtonian space; heat kernel; Poisson equation Renjin Jiang was partially supported by the Academy of Finland grants 120972 and 131477. 
$D u \cdot D u$ is comparable to the square of $\operatorname{Lip} u$, where

$$
\operatorname{Lip} u(x)=\limsup _{r \rightarrow 0} \sup _{d(x, y) \leq r} \frac{|u(x)-u(y)|}{r} .
$$

Having the above tools, the Lipschitz regularity of harmonic functions in $X$ is then considered in [15]. Let us first recall some notions. Let $\mu$ be a $Q$-regular measure on $X$ for some $Q \geq 1$, i.e., $\mu$ is Borel-regular and there exist constants $Q \geq 1$ and $C_{Q} \geq 1$ such that for every $x \in X$ and all $r>0$,

$$
C_{Q}^{-1} r^{Q} \leq \mu(B(x, r)) \leq C_{Q} r^{Q} .
$$

Let $\Omega \subseteq X$ be a domain. A function $u \in N_{\text {loc }}^{1,2}(X)$ is called Cheeger-harmonic in $\Omega$, if for all Lipschitz functions $\phi$ with compact support in $\Omega$,

$$
\int_{\Omega} D u(x) \cdot D \phi(x) d \mu(x)=0 .
$$

The following theorem was established in [15].

Theorem 1.1. Let $Q>1$ and suppose that $(X, d, \mu)$ supports a 2-Poincaré inequality. Furthermore, assume that there exist constants $C>0$ and $t_{0}>0$ such that for each $0<t<t_{0}$ and every $g \in N^{1,2}(X)$,

$$
\begin{aligned}
\int_{X} g(y)^{2} p(t, x, y) d \mu(y) \leq & \left(2 t+C t^{2}\right) \int_{X}|D g(y)|^{2} p(t, x, y) d \mu(y) \\
& +\left(\int_{X} g(y) p(t, x, y) d \mu(y)\right)^{2}
\end{aligned}
$$

for almost every $x \in X$. If $u$ is Cheeger-harmonic in $\Omega$, where $\Omega \subset X$ is a domain, then $u$ is locally Lipschitz continuous in $\Omega$.

Above, $p(t, x, y)$ refers to the heat kernel associated to the Dirichlet form $\int_{X} D f(x) \cdot D g(x) d \mu(x)$; see Subsection 2.2 below.

It is well known that (1.1) can be deduced from the logarithmic Sobolev inequality

$$
\begin{gathered}
\int_{X} f(x)^{2} \log \left(\frac{f(x)^{2}}{\|f\|_{L^{2}\left(X, p\left(t, x_{0}, x\right) d \mu\right)}^{2}}\right) p\left(t, x_{0}, x\right) d \mu(x) \\
\leq\left(4 t+2 C t^{2}\right) \int_{X}|\nabla f(x)|^{2} p\left(t, x_{0}, x\right) d \mu(x)
\end{gathered}
$$

see, for example, [1].

We remark that the authors in [15] gave several examples to show that: (i) in the abstract settings, harmonic functions may not be smooth and local Lipschitz continuity may be the best possible regularity; (ii) doubling of $\mu$ is not enough to guarantee the local Lipschitz continuity of harmonic functions and it is natural to consider an Ahlfors $Q$-regular measure; (iii) even when the Poincaré inequality and Ahlfors $Q$-regularity hold, harmonic functions may still not be locally Lipschitz continuous; hence a Sobolev-Poincaré inequality (1.1) is needed. 
Inspired by [15], in this paper, we work on the Lipschitz regularity of solutions of the Poisson equations in metric spaces. Let $\Omega \subseteq X$ be a domain. A Sobolev function $u \in N_{\text {loc }}^{1,2}(X)$ is called a solution of the equation $\Delta u=f$ in $\Omega$, if

$$
-\int_{\Omega} D u(x) \cdot D \phi(x) d \mu(x)=\int_{\Omega} f(x) \phi(x) d \mu(x), \quad \forall \phi \in N_{0}^{1,2}(\Omega) .
$$

Theorem 1.2. Let $Q \geq 1$ and suppose that $(X, d, \mu)$ supports a 2-Poincaré inequality and that (1.1) holds. Let $u \in N_{\text {loc }}^{1,2}(X)$ satisfy $\Delta u=f$ in $\Omega$, where $\Omega \subseteq X$ is a domain and $f \in L^{p}(\Omega)$. If $p>Q$, then $u$ is locally Lipschitz continuous in $\Omega$.

Notice that in our abstract cases, we can only define the first order derivative (Cheeger derivative), and the space $X$ does not have any geometric structure. Thus many classical methods are not available for Theorem 1.2. In this paper, we inherit the method investigated in [5] and used by Koskela et al [15] to study the Lipschitz regularity of harmonic functions in metric measure spaces. The method involves the abstract theory of Dirichlet forms and the heat equation. It is worth pointing out that the method used in $[5,15]$ can not be directly adapted to our setting: we need to modify the definition of a crucial functional $J(t)$; see $[5,1.1 .5]$, [15, p.167] and (3.1) below.

The paper is organized as follows. In Section 2, we give some basic notation and notions for Newtonian spaces, the Cheeger derivative and Dirichlet forms. Several auxiliary results are also given. Section 3 is devoted to the proof of Theorem 1.2. In Section 4, we discuss some situations where the Sobolev-Poincaré inequality (1.1) can be verified.

Finally, we make some conventions. Throughout the paper, we denote by $C$ a positive constant which is independent of the main parameters, but which may vary from line to line. We also use $C_{\gamma, \beta, \cdots}$ to denote a positive constant depending on the indicated parameters $\gamma, \beta, \cdots$.

\section{Preliminaries}

In this section, we give some basic notation and notions and several auxiliary results.

\subsection{Cheeger derivative in metric spaces}

Let us first recall the definition of Newtonian spaces on $X$ following [18]. Notice that the measure $\mu$ is only required to be doubling in [18] and Ahlfors $Q$-regular measures are always doubling measures.

The Newtonian space $N^{1, p}(X)$ is defined to be the space of all $p$-integrable (equivalence classes of) functions for which there exists a $p$-integrable upper gradient. If $u \in N^{1, p}(X)$, then we define its pseudonorm by

$$
\|u\|_{N^{1, p}(X)}:=\|u\|_{L^{p}(X)}+\inf _{g}\|g\|_{L^{p}(X)} .
$$

where the infimum is taken over all upper gradients of $u$. Further, if $(X, d, \mu)$ supports the $p$ Poincare inequality, then it is proved in [18] that the set of all Lipschitz functions are dense in $N^{1, p}(X)$. It is then natural to define the Newtonian spaces on open subsets and local Newtonian spaces $N_{\text {loc }}^{1, p}(X)$. The Sobolev spaces with zero boundary values on metric spaces were studied in 
[14]. For a domain $\Omega \subset X$, following [14], we define the Newtonian space with zero boundary values $N_{0}^{1, p}(X)$ to be the space of $u \in N^{1, p}(X)$ for which $u \chi_{X \backslash \Omega}$ vanishes $p$-quasi everywhere. Recall that a property holds $p$-quasi everywhere, if it holds except of a set of $p$-capacity zero.

Cheeger [6] introduced Sobolev spaces in metric spaces by using upper gradients in a different way, but it was proved in [18] that the Sobolev spaces in [6] coincide with the corresponding Newtonian spaces for $p>1$. The following theorem established in [6] provides us with a differential structure on metric spaces.

Theorem 2.1. Assume that $(X, d, \mu)$ supports a weak p-Poincaré inequality for some $p>1$ and that $\mu$ is doubling. Then there exists $N>0$, depending only on the doubling constant and the constants in the Poincaré inequality, such that the following holds. There exists a countable collection of measurable sets $U_{\alpha}, \mu\left(U_{\alpha}\right)>0$ for all $\alpha$, and Lipschitz functions $X_{1}^{\alpha}, \cdots, X_{k(\alpha)}^{\alpha}: U_{\alpha} \rightarrow \mathbb{R}$, with $1 \leq k(\alpha) \leq N$ such that $\mu\left(X \backslash \cup_{\alpha=1}^{\infty} U_{\alpha}\right)=0$, and for all $\alpha$ and $X_{1}^{\alpha}, \cdots, X_{k(\alpha)}^{\alpha}$ the following holds: for $f: X \rightarrow R$ Lipschitz, there exist $V_{\alpha}(f) \subseteq U_{\alpha}$ such that $\mu\left(U_{\alpha} \backslash V_{\alpha}(f)\right)=0$, and Borel functions $b_{1}^{\alpha}(x, f), \cdots, b_{k(\alpha)}^{\alpha}(x, f)$ of class $L^{\infty}$ such that if $x \in V_{\alpha}$, then

$$
\operatorname{Lip}\left(f-a_{1} X_{1}^{\alpha}-\cdots-a_{k(\alpha)} X_{k(\alpha)}^{\alpha}\right)(x)=0
$$

if and only if $\left(a_{1}, \cdots, a_{k(\alpha)}\right)=\left(b_{1}^{\alpha}(x, f), \cdots, b_{k(\alpha)}^{\alpha}(x, f)\right)$. Moreover, for almost every $x \in U_{\alpha_{1}} \cap U_{\alpha_{2}}$, the "coordinate functions" $X_{i}^{\alpha_{2}}$ are linear combinations of the $X_{i}^{\alpha_{1}}$ 's.

By Theorem 2.1, for each Lipschitz function $u$ we can assign a derivative $D u$, which maps $V_{\alpha} \subset X$ into $\mathbb{R}^{k(\alpha)}$, where $\alpha \in \mathbb{N}$ and $V_{\alpha}, k(\alpha)$ are as above. Moreover, the differential operator $D$, and hence the Laplacian operator $\Delta$, depend on the charts chosen.

Cheeger further showed that the differential operator $D$ can be extended to all functions in the corresponding Sobolev spaces, which coincide with the Newtonian spaces $N^{1, p}(X)$. A useful fact is that the Cheeger derivative satisfies the Leibniz rule, i.e.,

$$
D(u v)(x)=u(x) D v(x)+v(x) D u(x) .
$$

Moreover, the Euclidean norm $|D u|$ of $D u$ is comparable to Lip $u$.

We now introduce several useful inequalities. Recall that $(X, \mu)$ is an Ahlfors $Q$-regular space and supports a weak 2-Poincaré inequality. Then there exists a positive constant $C$, only depending on $Q, C_{P}$ and $C_{Q}$, such that for all $u \in N_{0}^{1,2}(B(x, r))$

$$
\left(f_{B(x, r)}|u(y)|^{s} d \mu(y)\right)^{1 / s} \leq C r\left(f_{B(x, r)}|D u(y)|^{2} d \mu(y)\right)^{1 / 2},
$$

where $s=\frac{2 Q}{Q-2}$ if $Q>2$, and $s>2$ is arbitrary if $Q \leq 2$; see [4, (k)] and also [3, 12, 16]. Notice that a (2,2)-Poincaré inequality is required in [4], but this is equivalent to the 2-Poincaré inequality under our assumptions; see [11,12].

Lemma 2.1. Let $p>Q$. There exists $C>0$ such that for all $u \in N_{\operatorname{loc}}^{1,2}(X)$ that satisfy $\Delta u=f$ in $B\left(x_{0}, 2 r\right)$, where $f \in L_{\mathrm{loc}}^{p}(X)$ and $B\left(x_{0}, 2 r\right) \subset \subset X$ it holds

$$
\sup _{B\left(x_{0}, r\right)}|u| \leq C\left[r^{-Q / 2}\|u\|_{L^{2}\left(B\left(x_{0}, 2 r\right)\right)}+r^{2-Q / p}\|f\|_{L^{p}\left(B\left(x_{0}, 2 r\right)\right)}\right] .
$$


Proof. When $Q \geq 2$, by [4, p.131], there exists $\widetilde{u} \in N_{0}^{1,2}\left(B\left(x_{0}, 2 r\right)\right)$ such that $\Delta \widetilde{u}=f$ in $B\left(x_{0}, 2 r\right)$. Then from [4, Theorem 4.1], we deduce that

$$
\sup _{B\left(x_{0}, 2 r\right)}|\widetilde{u}| \leq C r^{2} \mu\left(B\left(x_{0}, r\right)\right)^{-1 / p}\|f\|_{L^{p}\left(B\left(x_{0}, 2 r\right)\right)} .
$$

Now the fact that $u-\widetilde{u}$ is Cheeger-harmonic in $B\left(x_{0}, 2 r\right)$ and [4, Theorem 5.4] imply that

$$
\sup _{B\left(x_{0}, r\right)}|u-\widetilde{u}| \leq C r^{-Q / 2}\|u-\widetilde{u}\|_{L^{2}\left(B\left(x_{0}, 2 r\right)\right)},
$$

which together with (2.3) implies that (2.2) holds.

When $Q \in[1,2)$, we choose a Lipschitz function $\phi$ on $X$ such that $\phi \equiv 1$ on $B\left(x_{0}, r\right)$, supp $\phi \subseteq$ $B\left(x_{0}, 2 r\right)$ and $|D \phi| \leq C / r$. Then it is easy to see that $u \phi \in N_{0}^{1,2}\left(B\left(x_{0}, 2 r\right)\right)$. By [12, Theorem 5.1], the Hölder inequality and the Young inequality, we obtain that for every $x \in B\left(x_{0}, 2 r\right)$,

$$
\begin{aligned}
|(u \phi)(x)|^{2} & \leq C r^{2} f_{B\left(x_{0}, 2 r\right)}|D(u \phi)(y)|^{2} d \mu(y) \\
& =C r^{2} f_{B\left(x_{0}, 2 r\right)}\left[D u(y) \cdot D\left(u \phi^{2}\right)(y)+|u(y) D \phi(y)|^{2}\right] d \mu(y) \\
& \leq C r^{2} f_{B\left(x_{0}, 2 r\right)}\left[f(y) u(y) \phi(y)^{2}+\frac{u(y)^{2}}{r^{2}}\right] d \mu(y) \\
& \left.\leq \sup _{B\left(x_{0}, 2 r\right)}|u \phi|\right) \frac{C r^{2}\|f\|_{L^{p}\left(B\left(x_{0}, 2 r\right)\right)}}{\mu\left(B\left(x_{0}, 2 r\right)\right)^{1 / p}}+\frac{C\|u\|_{L^{2}\left(B\left(x_{0}, 2 r\right)\right)}^{2}}{\mu\left(B\left(x_{0}, 2 r\right)\right)} \\
& \leq \frac{1}{2} \sup _{B\left(x_{0}, 2 r\right)}|u \phi|^{2}+\frac{C r^{4}\|f\|_{L^{p}\left(B\left(x_{0}, 2 r\right)\right)}^{2}}{\mu\left(B\left(x_{0}, 2 r\right)\right)^{2 / p}}+\frac{C\|u\|_{L^{2}\left(B\left(x_{0}, 2 r\right)\right)}^{2}}{\mu\left(B\left(x_{0}, 2 r\right)\right)} .
\end{aligned}
$$

Hence

$$
\sup _{B\left(x_{0}, r\right)}|u| \leq \sup _{B\left(x_{0}, 2 r\right)}|(u \phi)| \leq \frac{C r^{2}\|f\|_{L^{p}\left(B\left(x_{0}, 2 r\right)\right)}}{\mu\left(B\left(x_{0}, 2 r\right)\right)^{1 / p}}+\frac{C\|u\|_{L^{2}\left(B\left(x_{0}, 2 r\right)\right)}}{\mu\left(B\left(x_{0}, 2 r\right)\right)^{1 / 2}}
$$

which implies that (2.2) holds, and hence completes the proof of Lemma 2.1.

We also need the following Caccioppoli inequality.

Lemma 2.2. Let $p>Q$. There exists $C>0$ such that for all $0<r<R$ and $u \in N_{\text {loc }}^{1,2}(X)$ that satisfy $\Delta u=f$ in $B\left(x_{0}, 2 R\right)$ with $f \in L_{\mathrm{loc}}^{p}(X)$ and $B\left(x_{0}, 2 R\right) \subset \subset X$ it holds

$$
\int_{B\left(x_{0}, r\right)}|D u(x)|^{2} d \mu(x) \leq C R^{2+Q\left(1-\frac{2}{p}\right)}\|f\|_{L^{p}\left(B\left(x_{0}, 2 R\right)\right)}^{2}+\frac{C}{(R-r)^{2}}\|u\|_{L^{2}\left(B\left(x_{0}, 2 R\right)\right)}^{2} .
$$

Proof. Choose a Lipschitz function $\phi$ such that $\phi=1$ on $B\left(x_{0}, r\right)$, supp $\phi \subset B\left(x_{0}, R\right)$ and $|D \phi| \leq$ $\frac{C}{R-r}$. Thus, $u \phi^{2} \in N_{0}^{1,2}\left(B\left(x_{0}, R\right)\right)$. By the Leibniz rule and (1.3), we have

$$
\int_{X} D u(x) \cdot D\left(u \phi^{2}\right)(x) d \mu(x)=\int_{X} \phi(x)^{2}|D u(x)|^{2}+2 u(x) \phi(x) D u(x) \cdot D \phi(x) d \mu(x)
$$




$$
=-\int_{X} f(x) u(x) \phi(x)^{2} d \mu(x) .
$$

Applying the Hölder inequality, the Young inequality and Lemma 2.1, we conclude that

$$
\begin{aligned}
& \int_{X} \phi(x)^{2}|D u(x)|^{2} d \mu(x) \\
& \leq \int_{X}|f(x) u(x)| \phi(x)^{2} d \mu(x)-\int_{X} 2 u(x) \phi(x) D u(x) \cdot D \phi(x) d \mu(x) \\
& \leq \int_{B\left(x_{0}, R\right)}|f(x) u(x)| d \mu(x)+\frac{1}{2}\left\|\phi\left|D u\left\|_{L^{2}(X)}^{2}+8\right\| u\right| D \phi\right\|_{L^{2}(X)}^{2} \\
& \leq C R^{2+Q\left(1-\frac{2}{p}\right)}\|f\|_{L^{p}\left(B\left(x_{0}, 2 R\right)\right)}^{2}+C R^{Q\left(\frac{1}{2}-\frac{1}{p}\right)}\|f\|_{L^{p}\left(B\left(x_{0}, R\right)\right)}\|u\|_{L^{2}\left(B\left(x_{0}, 2 R\right)\right)} \\
& \quad+\frac{1}{2}\|\phi \mid D u\|_{L^{2}(X)}^{2}+\frac{C}{(R-r)^{2}}\|u\|_{L^{2}\left(B\left(x_{0}, R\right)\right)}^{2} \\
& \leq C R^{2+Q\left(1-\frac{2}{p}\right)}\|f\|_{L^{p}\left(B\left(x_{0}, 2 R\right)\right)}^{2}+\frac{1}{2}\|\phi \mid D u\|_{L^{2}(X)}^{2}+\frac{C}{(R-r)^{2}}\|u\|_{L^{2}\left(B\left(x_{0}, 2 R\right)\right)}^{2}
\end{aligned}
$$

which completes the proof of Lemma 2.2.

\subsection{Dirichlet forms and heat kernels}

Having the Sobolev spaces $N^{1, p}(X)$ and the differential operator $D$, we now turn to the Dirichlet forms on $(X, d, \mu)$. Define the bilinear form $\mathscr{E}$ by

$$
\mathscr{E}(f, g)=\int_{X} D f(x) \cdot D g(x) d \mu(x)
$$

with the domain $D(\mathscr{E})=N^{1,2}(X)$. Then $\mathscr{E}$ is symmetric and closed. Corresponding to such a form there exists an infinitesimal generator $A$ which acts on a dense subspace $D(A)$ of $N^{1,2}(X)$ so that for all $f \in D(A)$ and each $g \in N^{1,2}(X)$,

$$
\int_{X} g(x) A f(x) d \mu(x)=-\mathscr{E}(g, f) .
$$

Now let us recall several auxiliary results established in [15].

Lemma 2.3. If $u, v \in N^{1,2}(X)$, and $\phi \in N^{1,2}(X)$ is a bounded Lipschitz function, then

$$
\mathscr{E}(\phi, u v)=\mathscr{E}(\phi u, v)+\mathscr{E}(\phi v, u)-2 \int_{X} \phi D u(x) \cdot D v(x) d \mu(x)
$$

Moreover, if $u, v \in \mathscr{D}(A)$, then we can unambiguously define the $L^{1}$-function $A(u v)$ by setting

$$
A(u v)=u A v+v A u+2 D u \cdot D v .
$$

Also, associated with the Dirichlet form $\mathscr{E}$, there is a semigroup $\left\{T_{t}\right\}_{t>0}$, acting on $L^{2}(X)$, with the following properties (see [8, Chapter 1]):

1. $T_{t} \circ T_{s}=T_{t+s}, \forall t, s>0$, 
2. $\int_{X}\left|T_{t} f(x)\right|^{2} d \mu(x) \leq \int_{X} f(x)^{2} d \mu(x), \forall f \in L^{2}(X, \mu)$ and $\forall t>0$,

3. $T_{t} f \rightarrow f$ in $L^{2}(X, \mu)$ when $t \rightarrow 0$,

4. if $f \in L^{2}(X, \mu)$ satisfies $0 \leq f \leq C$, then $0 \leq T_{t} f \leq C$ for all $t>0$,

5. if $f \in \mathscr{D}(A)$, then $\frac{1}{t}\left(T_{t} f-f\right) \rightarrow A f$ in $L^{2}(X, \mu)$ as $t \rightarrow 0$, and

6. $A T_{t} f=\frac{\partial}{\partial_{t}} T_{t} f, \forall t>0$ and $\forall f \in L^{2}(X, \mu)$.

A measurable function $p: \mathbb{R} \times X \times X \rightarrow[0, \infty]$ is said to be a heat kernel on $X$ if

$$
T_{t} f(x)=\int_{X} f(y) p(t, x, y) d \mu(y)
$$

for every $f \in L^{2}(X, \mu)$ and all $t \geq 0$, and $p(t, x, y)=0$ for every $t<0$. Let the measure on $X$ be doubling and support a 2-Poincaré inequality. Sturm ([20]) proved the existence of the heat kernel, and a Gaussian estimate for the heat kernel which in our settings reads as: there exist positive constants $C, C_{1}, C_{2}$ such that

$$
C^{-1} t^{-\frac{Q}{2}} e^{-\frac{d(x, y)^{2}}{C_{2} t}} \leq p(t, x, y) \leq C t^{-\frac{Q}{2}} e^{-\frac{d(x, y)^{2}}{C_{1} t}}
$$

Moreover, the heat kernel is proved in [19] to be a probability measure, i.e., for each $x \in X$ and $t>0$

$$
T_{t} 1(x)=\int_{X} p(t, x, y) d \mu(y)=1 .
$$

The following Lemma 2.4, Lemma 2.5 and Proposition 2.1 were established in [15] for $Q>1$. However, their proofs show that they also hold for all $Q \geq 1$. We omit the details here.

Lemma 2.4. Let $T>0$. Then for $\mu$-almost every $x \in X, D_{y} p(\cdot, x, \cdot) \in L^{2}([0, T] \times X)$ and there exists a positive constant $C_{T, x}$, depending on $T$ and $x$, such that

$$
\int_{0}^{T} \int_{X}\left|D_{y} p(t, x, y)\right|^{2} d \mu(y) d t \leq C_{T, x}
$$

Lemma 2.5. There exists $C>0$ such that for all $0<T<r^{3}<1$ and every $x \in X$ it holds

$$
\int_{0}^{T} \int_{B(x, 2 r) \backslash B(x, 2 r)}\left|D_{y} p(t, x, y)\right|^{2} d \mu(y) d t \leq e^{-C T^{-1 / 3}} .
$$

The following result shows that the heat kernel plays the role of a fundamental solution. Let us recall the definition of test functions in this subject. The test functions are continuous functions $\phi$ : $[0, T] \times X \rightarrow \mathbb{R}$ such that for every fixed $t>0, \phi(t, \cdot)=\phi_{t}(\cdot) \in N^{1,2}(X), D_{y} \phi(t, y) \in L^{2}([0, T] \times X)$, and for $\mu$-almost every $x \in X, \phi(\cdot, x)$ is absolutely continuous on $[0, T]$. Moreover, we assume that there is a constant $\delta_{0}=\delta_{0}(x)>0$ such that the following Hölder continuity property holds for $\phi$ and the "center point" $x$ of the heat kernel $p(\cdot, x, \cdot)$ : there exist $C$ and $\alpha>0$ such that for every $\delta<\delta_{0}$ and for all $(t, y) \in[0, \delta] \times B(x, \delta)$,

$$
|\phi(t, y)-\phi(0, x)| \leq C \delta^{\alpha}
$$


Proposition 2.1. There exists a constant $K$ such that for every test function $\phi_{t}(y)$ and every $x \in X$ it holds

$$
\begin{aligned}
\int_{0}^{T} \int_{X} \phi_{t}(y) A_{y} p(t, x, y) d \mu(y) d t & :=-\int_{0}^{T} \int_{X} D \phi_{t}(y) \cdot D_{y} p(t, x, y) d \mu(y) d t \\
& =\int_{0}^{T} \int_{X} \phi_{t}(y) \frac{\partial}{\partial t} p(t, x, y) d \mu(y) d t+K \phi_{0}(x) .
\end{aligned}
$$

\section{Proof of Theorem 1.2}

Proof. Our proof is developed from the proof of [15, Theorem 1]; see also [5]. The proof is quite long, so that we first describe the strategy. Let $u$ be a solution of (1.3) on a domain $\Omega \subseteq X$ and let $r \in(0,1)$ and $B=B\left(y_{0}, 6 r\right) \subset \subset \Omega$. Following [15], we want to bound $\left|D u\left(x_{0}\right)\right|$ for every $x_{0} \in B\left(y_{0}, r\right) \backslash A$, where $A$ is a set of measure zero depending on $u$ and the heat kernel.

Let $\phi$ be a Lipschitz function on $X$ such that $\phi \equiv 1$ on $B\left(x_{0}, r\right)$, supp $\phi \subseteq B\left(x_{0}, 2 r\right)$ and $|D \phi| \leq$ $C / r$. Now fix $T<r^{3}$ and for every $t \in(0, T)$, set

$$
w(t, x):=u(x) \phi(x)-T_{t}(u \phi)\left(x_{0}\right) .
$$

Then $D w\left(t, x_{0}\right)=D(u \phi)\left(x_{0}\right)=D u\left(x_{0}\right)$.

Let $\epsilon \in(0,1)$ be determined in the future. Now, for $t \in(0, T]$, let

$$
\begin{aligned}
J(t):= & \frac{1+t^{\epsilon}}{t} \int_{0}^{t} \int_{X}|D w(s, x)|^{2} p\left(s, x_{0}, x\right) d \mu(x) d s \\
& +\frac{1+t^{\epsilon}}{t} \int_{0}^{t} \int_{X} w(s, x) \phi(x) A u(x) p\left(s, x_{0}, x\right) d \mu(x) d s .
\end{aligned}
$$

From the following proofs, we will see that $\epsilon$ needs to be chosen depending on $p, Q$, and that the term of $\epsilon$ plays a crucial role in proving the theorem.

We will prove the following results.

Proposition 3.1. There exists a positive constant $C_{T, r}$, depending on $T, r$, such that

$$
J(T) \leq C_{T, r}\left(\|u\|_{L^{2}\left(B\left(x_{0}, 4 r\right)\right)}+\|f\|_{L^{p}\left(B\left(x_{0}, 4 r\right)\right)}\right)^{2} .
$$

Proposition 3.2. There exists a positive constant $C_{T, r}$, depending on $T, r$, such that

$$
\int_{0}^{T} \frac{d}{d t} J(t) d t \geq-C_{T, r}\left(\|u\|_{L^{2}\left(B\left(x_{0}, 4 r\right)\right)}+\|f\|_{L^{p}\left(B\left(x_{0}, 4 r\right)\right)}\right)^{2}
$$

Proposition 3.3. For almost every $x_{0} \in B\left(y_{0}, r\right)$ it holds $\lim _{t \rightarrow 0^{+}} J(t)=\left|D u\left(x_{0}\right)\right|$.

Combining Propositions 3.1, 3.2 and 3.3, we finally obtain

$$
\begin{aligned}
\left|D(u)\left(x_{0}\right)\right|^{2} & =J(T)-\int_{0}^{T} \frac{d}{d t} J(t) d t \\
& \leq C_{T, r}\left(\|u\|_{L^{2}\left(B\left(x_{0}, 4 r\right)\right)}+\|f\|_{L^{p}\left(B\left(x_{0}, 4 r\right)\right)}\right)^{2},
\end{aligned}
$$


for almost every $x_{0} \in B\left(y_{0}, r\right)$.

Now for all $x, y \in B\left(y_{0}, r\right) \subseteq \Omega$ with $B\left(y_{0}, 6 r\right) \subset \subset \Omega$, let $B_{1}=B(x, d(x, y))$ and $B_{-1}=$ $B(y, d(x, y))$. For $j \geq 1$, set $B_{j}=2^{-1} B_{i-1}$ and $B_{-j}=2^{-1} B_{-j+1}$ inductively. Further, if $x, y$ are Lebesgue points of $u$, then

$$
|u(x)-u(y)| \leq \sum_{j=-\infty}^{\infty}\left|u_{B_{j}}-u_{B_{j+1}}\right|
$$

where for each $j$, the Poincaré inequality yields

$$
\begin{aligned}
\left|u_{B_{j}}-u_{B_{j+1}}\right| & \leq C \operatorname{diam}\left(2 B_{j}\right)\left(f_{2 B_{j}}|D u(x)|^{2} d \mu(x)\right)^{1 / 2} \\
& \leq C_{T, r} \operatorname{diam}\left(2 B_{j}\right)\left(\|u\|_{L^{2}\left(B\left(y_{0}, 6 r\right)\right)}+\|f\|_{L^{p}\left(B\left(y_{0}, 6 r\right)\right)}\right) .
\end{aligned}
$$

Hence, we obtain

$$
|u(x)-u(y)| \leq C_{T, r}\left(\|u\|_{L^{2}\left(B\left(y_{0}, 6 r\right)\right)}+\|f\|_{L^{p}\left(B\left(y_{0}, 6 r\right)\right)}\right) d(x, y),
$$

for almost all $x, y \in B\left(y_{0}, r\right)$. Then $u$ can be extended to a locally Lipschitz continuous function on $\Omega$, which completes the proof of Theorem 1.2.

Let us prove the Propositions.

Proof of Proposition 3.1. Since $w(t, x)=u(x) \phi(x)-T_{t}(u \phi)\left(x_{0}\right)$, we have

$$
|D(u \phi)|^{2}=|D w|^{2}=\frac{1}{2} A w^{2}-w(u A \phi+\phi A u+2 D u \cdot D \phi)
$$

in the weak sense of measures. Also, in what follows we extend $A$ formally to all of $N^{1,2}(X)$ by defining

$$
\int_{X} v(x) A u(x) d \mu(x)=-\int_{X} D v(x) \cdot D u(x) d \mu(x)=\int_{X} A v(x) u(x) d \mu(x) .
$$

Moreover, we set $m(t)=T_{t}(u \phi)\left(x_{0}\right)$. Then $\frac{\partial}{\partial t} w^{2}=2 w \frac{\partial}{\partial t} w=-2 w m^{\prime}(t)$, which further implies that

$$
|D w|^{2}=\frac{1}{2}\left(A+\frac{\partial}{\partial t}\right) w^{2}-w\left(u A \phi+\phi A u+2 D u \cdot D \phi-m^{\prime}(t)\right)
$$

in the weak sense of measures. Thus, we obtain

$$
\begin{aligned}
\int_{0}^{t} & \int_{X}\left[|D w(s, x)|^{2}+w(s, x) \phi(x) A u(x)\right] p\left(s, x_{0}, x\right) d \mu(x) d s \\
= & \frac{1}{2} \int_{0}^{t} \int_{X}\left(A+\frac{\partial}{\partial s}\right) w^{2}(s, x) p\left(s, x_{0}, x\right) d \mu(x) d s \\
& -\int_{0}^{t} \int_{X} w(s, x)\left[u(x) A \phi(x)+2 D u(x) \cdot D \phi(x)-m^{\prime}(s)\right] p\left(s, x_{0}, x\right) d \mu(x) d s .
\end{aligned}
$$

Recall that for each $s>0$ and $x_{0} \in X, T_{s}(1)\left(x_{0}\right)=\int_{X} p\left(s, x_{0}, x\right) d \mu(x)=1$. We then have

$$
\int_{0}^{t} \int_{X} w(s, x) m^{\prime}(s) p\left(s, x_{0}, x\right) d \mu(x) d s=\int_{0}^{t} m^{\prime}(s) T_{s}(u \phi)\left(x_{0}\right)\left(1-T_{s}(1)\left(x_{0}\right)\right) d s=0 .
$$


Now applying integration by parts and using (3.2), we obtain

$$
\begin{aligned}
\int_{0}^{t} & \int_{X}\left(A+\frac{\partial}{\partial s}\right) w^{2}(s, x) p\left(s, x_{0}, x\right) d \mu(x) d s \\
= & \int_{0}^{t} \int_{X} w^{2}(s, x)\left(A-\frac{\partial}{\partial s}\right) p\left(s, x_{0}, x\right) d \mu(x) \\
& +\int_{X} w^{2}(t, x) p\left(t, x_{0}, x\right) d \mu(x)-\int_{X} w^{2}(0, x) p\left(0, x_{0}, x\right) d \mu(x) .
\end{aligned}
$$

At this point, we want to use Proposition 2.1 with $\phi(t, x)=w^{2}(t, x)$. By Lemma 2.1 and $u \in$ $N_{\text {loc }}^{1,2}(X)$, we have $D_{x}\left(w^{2}(t, x)\right)=2 w(t, x) D(u \phi)(x) \in L^{2}([0, T] \times X)$. The property (6) of our semigroup guarantees the continuity of $w^{2}(t, x)$ on $[0, T]$. Since $w^{2}(t, x)$ may equal to a constant outside $B\left(x_{0}, 2 r\right)$, it may not be in $L^{2}(X)$, but we always have $D\left(w^{2}(t, \cdot)\right) \in L^{2}(X)$ which is enough for us to use Proposition 2.1. The only thing left is to verify the Hölder continuity of $w^{2}(t, x)$.

For $p>Q \geq 2$, by [4, Theorem 5.13], we see that $u$ is locally Hölder continuous; while for $1 \leq Q<2$, since $u \phi \in N^{1,2}(X)$, by [18, Theorem 5.1], $u \phi$ is Hölder continuous with exponent $1-\frac{Q}{2}$. More precisely, for almost all $x, y \in B$, we have

$$
|u(x)-u(y)| \leq C_{r}\left[\|u\|_{L^{2}\left(B\left(x_{0}, 4 r\right)\right)}+\|g\|_{L^{p}\left(B\left(x_{0}, 4 r\right)\right)}\right] d(x, y)^{\delta} .
$$

Notice here that, when $Q<2, \delta=1-\frac{Q}{2}$. In what follows, for simplicity, we define

$$
C(u, f):=\|u\|_{L^{2}\left(B\left(x_{0}, 4 r\right)\right)}+\|f\|_{L^{p}\left(B\left(x_{0}, 4 r\right)\right)} .
$$

In the following proof, we will repeatedly use the fact that for fixed $\beta, \gamma \in(0, \infty), t^{\beta} e^{-t^{\gamma}}$ and $t^{-\beta} e^{-t^{-\gamma}}$ are bounded on $(0, \infty)$. Let us now show that (2.7) holds for $w$. By the local Hölder continuity of $u \phi$ and (2.5), we have

$$
\begin{aligned}
& |w(t, x)|=\left|u(x) \phi(x)-T_{t}(u \phi)\left(x_{0}\right)\right| \\
& \quad=\left|u(x) \phi(x)-u\left(x_{0}\right) \phi\left(x_{0}\right)+u\left(x_{0}\right) \phi\left(x_{0}\right)-T_{t}(u \phi)\left(x_{0}\right)\right| \\
& \quad \leq C C(u, f) d\left(x, x_{0}\right)^{\delta}+\int_{X}\left|u\left(x_{0}\right) \phi\left(x_{0}\right)-u(x) \phi(x)\right| p\left(t, x_{0}, x\right) d \mu(x) \\
& \quad \leq C C(u, f)\left\{d\left(x, x_{0}\right)^{\delta}+\int_{X} d\left(x, x_{0}\right)^{\delta} t^{-\frac{Q}{2}} e^{-\frac{d\left(x, x_{0}\right)^{2}}{2 C_{1} t}} e^{-\frac{d\left(x, x_{0}\right)^{2}}{2 C_{1} t}} d \mu(x)\right\} \\
& \quad \leq C C(u, f)\left\{d\left(x, x_{0}\right)^{\delta}+t^{\delta / 2} \int_{X} p\left(l t, x_{0}, x\right) d \mu(x)\right\} \\
& \quad \leq C C(u, f)\left(d\left(x, x_{0}\right)^{\delta}+t^{\delta / 2}\right)
\end{aligned}
$$

where $l=\frac{2 C_{1}}{C_{2}}$. Now let $\gamma \in(0, T]$ and $(t, x) \in[0, \gamma] \times B\left(x_{0}, \gamma\right)$. Then by (3.4) and the fact that $w\left(0, x_{0}\right)=0$, we see that

$$
\left|w(t, x)-w\left(0, x_{0}\right)\right| \leq C C(u, f)\left(d\left(x, x_{0}\right)^{\delta}+t^{\delta / 2}\right) \leq C C(u, f) \gamma^{\delta / 2} .
$$

Thus, this allows us to use Proposition 2.1 to obtain

$$
\int_{0}^{t} \int_{X}\left(A+\frac{\partial}{\partial s}\right) w^{2}(s, x) p\left(s, x_{0}, x\right) d \mu(x) d s
$$




$$
=K w^{2}\left(0, x_{0}\right)+\int_{X} w^{2}(t, x) p\left(t, x_{0}, x\right) d \mu(x)-\int_{X} w^{2}(0, x) p\left(0, x_{0}, x\right) d \mu(x) .
$$

Using (2.6) gives

$$
\begin{aligned}
\int_{X} w^{2}(0, x) p\left(0, x_{0}, x\right) d \mu(x) & =\lim _{s \rightarrow 0^{+}} \int_{X} w^{2}(s, x) p\left(s, x_{0}, x\right) d \mu(x) \\
& =\lim _{s \rightarrow 0^{+}}\left[T_{s}\left((u \phi)^{2}\right)\left(x_{0}\right)-\left(T_{s}(u \phi)\left(x_{0}\right)\right)^{2}\right]=0,
\end{aligned}
$$

which together with the fact $w\left(0, x_{0}\right)=0$ yields

$$
\int_{0}^{t} \int_{X}\left(A+\frac{\partial}{\partial s}\right) w^{2}(s, x) p\left(s, x_{0}, x\right) d \mu(x) d s=\int_{X} w^{2}(t, x) p\left(t, x_{0}, x\right) d \mu(x) .
$$

We now estimate the second term in (3.3). Recall that $\phi=1$ on $B\left(x_{0}, r\right)$ and $\operatorname{supp} \phi \subseteq B\left(x_{0}, 2 r\right)$. By Lemma 2.1, Lemma 2.2, Lemma 2.5 and the Hölder inequality, we obtain

$$
\begin{aligned}
& \left|\int_{0}^{t} \int_{X} w(s, x) u(x) A \phi(x) p\left(s, x_{0}, x\right) d \mu(x) d s\right| \\
& =\left|\int_{0}^{t} \int_{X} D\left(w(s, x) u(x) p\left(s, x_{0}, x\right)\right) \cdot D \phi(x) d \mu(x) d s\right| \\
& \leq\left|\int_{0}^{t} \int_{X} w(s, x) u(x) D p\left(s, x_{0}, x\right) \cdot D \phi(x) d \mu(x) d s\right| \\
& \quad+\left|\int_{0}^{t} \int_{X} w(s, x) p\left(s, x_{0}, x\right) D u(x) \cdot D \phi(x) d \mu(x) d s\right| \\
& \quad+\left|\int_{0}^{t} \int_{X} u(x) p\left(s, x_{0}, x\right) D w(s, x) \cdot D \phi(x) d \mu(x) d s\right| \\
& \leq C_{r}\|u\|_{L^{\infty}\left(B\left(x_{0}, 2 r\right)\right)}^{2}\left(\int_{0}^{t} \int_{B\left(x_{0}, 2 r\right) \backslash B\left(x_{0}, r\right)}\left|D p\left(s, x_{0}, x\right)\right|^{2} d \mu(x) d s\right)^{1 / 2} \\
& \quad+C_{r} e^{-C t^{-\frac{1}{3}}}\|u\|_{L^{\infty}\left(B\left(x_{0}, 2 r\right)\right)}\left(\int_{B\left(x_{0}, 2 r\right) \backslash B\left(x_{0}, r\right)}|D u(x)|^{2} d \mu(x)\right)^{1 / 2} \\
& \leq C_{r} e^{-C t^{-\frac{1}{3}}} C(u, f)^{2} .
\end{aligned}
$$

Similarly we have

$$
\left|\int_{0}^{t} \int_{X} w(s, x) p\left(s, x_{0}, x\right) D u(x) \cdot D \phi(x) d \mu(x) d s\right| \leq C_{r} C(u, f)^{2} e^{-C t^{-\frac{1}{3}}} .
$$

Combining the estimates (3.5)-(3.7), by (3.3), we obtain that $t \in(0, T]$,

$$
\begin{aligned}
J(t) \leq & \frac{1+t^{\epsilon}}{2 t}\left|\int_{0}^{t} \int_{X}\left(A+\frac{\partial}{\partial s}\right) w^{2}(s, x) p\left(s, x_{0}, x\right) d \mu(x) d s\right| \\
& +\frac{1+t^{\epsilon}}{t} \mid \int_{0}^{t} \int_{X} w(s, x)\left[u(x) A \phi(x)+2 D u(x) \cdot D \phi(x) p\left(s, x_{0}, x\right) d \mu(x) d s \mid\right.
\end{aligned}
$$




$$
\leq \frac{1+t^{\epsilon}}{2 t} \int_{X} w^{2}(t, x) p\left(t, x_{0}, x\right) d \mu(x)+C_{r} e^{-C t^{-\frac{1}{3}}} C(u, f)^{2} .
$$

By letting $t=T$ and using Lemma 2.1, we obtain

$$
J(T) \leq C_{T}\|u \phi\|_{L^{\infty}(X)}^{2}+C_{T, r} C(u, f)^{2} \leq C_{T, r} C(u, f)^{2},
$$

which is the desired estimate, and hence completes the proof of Proposition 3.1.

Proof of Proposition 3.2. Let us now estimate the derivative $J^{\prime}(t)=\frac{d}{d t} J(t)$. By Lemma 2.4, for almost every $x_{0} \in X, D_{y} p\left(s, x_{0}, \cdot\right) \in L^{2}(X)$. From this together with the fact that for each fixed $s$, $w(s, \cdot), \phi, p\left(s, x_{0}, \cdot\right)$ are bounded functions, we see that $w \phi p \in N_{0}^{1,2}\left(B\left(x_{0}, 2 r\right)\right)$. Thus by (3.2), we obtain

$$
\begin{aligned}
& \int_{0}^{t} \int_{X} w(s, x) \phi(x) A u(x) p\left(s, x_{0}, x\right) d \mu(x) \\
& =-\int_{0}^{t} \int_{X} D\left(w(s, \cdot) \phi p\left(s, x_{0}, \cdot\right)\right)(x) \cdot D u(x) d \mu(x) \\
& =\int_{0}^{t} \int_{X} w(s, x) \phi(x) f(x) p\left(s, x_{0}, x\right) d \mu(x) .
\end{aligned}
$$

This and (3.8) further imply that

$$
\begin{aligned}
\frac{d}{d t} J(t)= & \left(-\frac{1}{t^{2}}-\frac{1-\epsilon}{t^{2-\epsilon}}\right) \frac{t}{1+t^{\epsilon}} J(t)+\frac{1+t^{\epsilon}}{t} \int_{X}|D w(t, x)|^{2} p\left(t, x_{0}, x\right) d \mu(x) \\
& +\frac{1+t^{\epsilon}}{t} \int_{X} w(t, x) \phi(x) f(x) p\left(t, x_{0}, x\right) d \mu(x) \\
\geq & \frac{1+(1-\epsilon) t^{\epsilon}}{t}\left(\int_{X}|D w(t, x)|^{2} p\left(t, x_{0}, x\right) d \mu(x)-\frac{1}{2 t} \int_{X} w^{2}(t, x) p\left(t, x_{0}, x\right) d \mu(x)\right) \\
& -C_{r} e^{-C t^{-\frac{1}{3}}} C(u, f)^{2}+\frac{\epsilon t^{\epsilon}}{t} \int_{X}|D w(t, x)|^{2} p\left(t, x_{0}, x\right) d \mu(x) \\
& +\frac{1+t^{\epsilon}}{t} \int_{X} w(t, x) \phi(x) f(x) p\left(t, x_{0}, x\right) d \mu(x) .
\end{aligned}
$$

The main difficulty left is to estimate the last term in (3.10). To this end, we now decompose our proof into two different cases: (i) $Q \geq 2$ and (ii) $Q \in[1,2)$. From the following proof, we will see that the term $t^{\epsilon}$ in (3.1) plays a key role in matching the two terms $\int_{X}|D w(t, x)|^{2} p\left(t, x_{0}, x\right) d \mu(x)$ and $\frac{1}{2 t} \int_{X} w^{2}(t, x) p\left(t, x_{0}, x\right) d \mu(x)$, which allows us to use (1.1).

Case (i) $Q \geq 2$. Recall that $\epsilon \in(0,1)$. Applying the Young inequality to the last term in (3.10) and choosing suitable constants, we obtain

$$
\begin{aligned}
\frac{d}{d t} J(t) \geq & \frac{1+(1-\epsilon) t^{\epsilon}}{t}\left(\int_{X}|D w(t, x)|^{2} p\left(t, x_{0}, x\right) d \mu(x)-\frac{1}{2 t} \int_{X} w^{2}(t, x) p\left(t, x_{0}, x\right) d \mu(x)\right) \\
& -C_{r} e^{-C t^{-\frac{1}{3}}} C(u, f)+\frac{\epsilon t^{\epsilon}}{t} \int_{X}|D w(t, x)|^{2} p\left(t, x_{0}, x\right) d \mu(x) \\
& -\frac{\epsilon t^{\epsilon}}{2 t^{2}} \int_{X} w^{2}(t, x) p\left(t, x_{0}, x\right) d \mu(x)-\frac{C_{T, \epsilon}}{t^{\epsilon}} \int_{X}(\phi(x) f(x))^{2} p\left(t, x_{0}, x\right) d \mu(x)
\end{aligned}
$$




$$
\begin{aligned}
\geq & \frac{1+t^{\epsilon}}{t}\left(\int_{X}|D w(t, x)|^{2} p\left(t, x_{0}, x\right) d \mu(x)-\frac{1}{2 t} \int_{X} w^{2}(t, x) p\left(t, x_{0}, x\right) d \mu(x)\right) \\
& -C_{r} e^{-C t^{-\frac{1}{3}}} C(u, f)-\frac{C_{T, \epsilon}}{t^{\epsilon}} \int_{X}(\phi(x) f(x))^{2} p\left(t, x_{0}, x\right) d \mu(x) .
\end{aligned}
$$

For each fixed $t \in(0, T)$, either

$$
\int_{X}|D w(t, x)|^{2} p\left(t, x_{0}, x\right) d \mu(x) \geq \frac{1}{2 t} \int_{X} w^{2}(t, x) p\left(t, x_{0}, x\right) d \mu(x)
$$

or

$$
\int_{X}|D w(t, x)|^{2} p\left(t, x_{0}, x\right) d \mu(x)<\frac{1}{2 t} \int_{X} w^{2}(t, x) p\left(t, x_{0}, x\right) d \mu(x) .
$$

In the first case, we have

$$
\frac{d}{d t} J(t) \geq-C_{r} e^{-C t^{-\frac{1}{3}}} C(u, f)-\frac{C_{T, \epsilon}}{t^{\epsilon}} \int_{X}(\phi(x) f(x))^{2} p\left(t, x_{0}, x\right) d \mu(x) .
$$

Let us consider the second case. By (2.7), (3.4) and the fact that $\frac{d\left(x, x_{0}\right)^{\delta}}{t^{\delta / 2}} e^{-\frac{\left|x-x_{0}\right|^{2}}{2 C_{1} t}}$ is bounded, we obtain

$$
\begin{aligned}
& \int_{X}|D w(t, x)|^{2} p\left(t, x_{0}, x\right) d \mu(x)<\frac{1}{2 t} \int_{X} w^{2}(t, x) p\left(t, x_{0}, x\right) d \mu(x) \\
& \leq C C(u, f)^{2} \frac{1}{2 t} \int_{X}\left(d\left(x, x_{0}\right)^{\delta}+t^{\delta / 2}\right)^{2} t^{-Q / 2} e^{-\frac{\left|x-x_{0}\right|^{2}}{C_{1} t}} d \mu(x) \\
& \leq C C(u, f)^{2} t^{\delta-1} \int_{X} p\left(l t, x_{0}, x\right) d \mu(x) \leq C C(u, f)^{2} t^{\delta-1},
\end{aligned}
$$

where $l=\frac{C_{1}}{2 C_{2}}$. The fact $w=u \phi-T_{t}(u \phi)\left(x_{0}\right)$ implies

$$
\begin{aligned}
\int_{X} w^{2}(t, x) p\left(t, x_{0}, x\right) d \mu(x)= & \int_{X}(u(x) \phi(x))^{2} p\left(t, x_{0}, x\right) d \mu(x) \\
& -\left(\int_{X} u(x) \phi(x) p\left(t, x_{0}, x\right) d \mu(x)\right)^{2} .
\end{aligned}
$$

Then, by (1.1) with $g$ replaced by $u \phi$, we obtain

$$
\begin{aligned}
\frac{d}{d t} J(t) \geq & -C\left(1+t^{\epsilon}\right) \int_{X}|D w(t, x)|^{2} p\left(t, x_{0}, x\right) d \mu(x) \\
& -C_{r} e^{-C t^{-\frac{1}{3}}} C(u, f)-\frac{C_{T}}{t^{\epsilon}} \int_{X}(\phi(x) f(x))^{2} p\left(t, x_{0}, x\right) d \mu(x) \\
\geq & -C_{T, r} C(u, f)^{2} t^{\delta-1}-\frac{C_{T, \epsilon}}{t^{\epsilon}} \int_{X}(\phi(x) f(x))^{2} p\left(t, x_{0}, x\right) d \mu(x) .
\end{aligned}
$$

Thus, from (3.11) and (3.12), we see that (3.12) holds in both cases. 
Since $p>Q \geq 2$, we may choose $\epsilon \in(0,1)$ such that $Q / p+\epsilon<1$. This together with the Hölder inequality implies that

$$
\begin{aligned}
& \int_{0}^{T} \frac{d}{d t} J(t) d t \\
& \geq-C_{T, r} C(u, f)^{2} \int_{0}^{T} t^{\delta-1} d t-\int_{0}^{T} \frac{C_{T}}{t^{\epsilon}} \int_{X}(\phi(x) f(x))^{2} p\left(t, x_{0}, x\right) d \mu(x) d t \\
& \geq-C_{T, r} C(u, f)^{2}\left[1+\int_{0}^{T} t^{-\epsilon}\left(\int_{X} p\left(t, x_{0}, x\right)^{\frac{p}{p-2}} d \mu(x)\right)^{1-\frac{2}{p}} d t\right] \\
& \geq-C_{T, r} C(u, f)^{2} \int_{0}^{T} t^{-\epsilon-\frac{Q}{p}}\left(\int_{X} p\left(t, x_{0}, x\right) d \mu(x)\right)^{1-\frac{2}{p}} d t \\
& \geq-C_{T, r} C(u, f)^{2}
\end{aligned}
$$

which completes the proof of the case $Q \in[2, \infty)$.

Case (ii) $Q \in[1,2)$. Let us first estimate the last term in (3.10). Let $\alpha \in\left(0, \frac{1}{2}\right)$ be fixed in what follows. Choose $\psi_{t}(x)$ to be a Lipschitz function on $X$ such that $\psi_{t}(x) \equiv 1$ on $B\left(x_{0}, t^{\alpha}\right)$, $\operatorname{supp} \psi_{t} \subseteq B\left(x_{0}, 2 t^{\alpha}\right)$ and $|D \phi| \leq C t^{-\alpha}$. Write

$$
\begin{aligned}
& \frac{1+t^{\epsilon}}{t}\left|\int_{X} w(t, x) \phi(x) f(x) p\left(t, x_{0}, x\right) d \mu(x)\right| \\
& \leq \frac{C_{T}}{t} \int_{B\left(x_{0}, t^{\alpha}\right)}\left|\psi_{t}(x) w(t, x) \phi(x) f(x) p\left(t, x_{0}, x\right)\right| d \mu(x) \\
& \quad+\frac{C_{T}}{t} \int_{\left(B\left(x_{0}, t^{\alpha}\right)\right) C}^{C}\left|w(t, x) \phi(x) f(x) p\left(t, x_{0}, x\right)\right| d \mu(x) \\
& =: \mathrm{H}_{1}+\mathrm{H}_{2} .
\end{aligned}
$$

Since $\alpha \in\left(0, \frac{1}{2}\right), t^{-1-\frac{Q}{2}} e^{-\frac{t^{2 \alpha-1}}{2 C_{1}}}$ is bounded on $(0, \infty)$. This, together with the Hölder inequality and (2.5) yields

$$
\begin{aligned}
\mathrm{H}_{2} & \leq \frac{C_{T}}{t}\|u\|_{L^{\infty}\left(B\left(x_{0}, 2 r\right)\right)} \int_{\left(B\left(x_{0}, t^{\alpha}\right)\right) C}|\phi(x) f(x)| t^{-\frac{Q}{2}} e^{-\frac{d\left(x, x_{0}\right)^{2}}{C_{1} t}} d \mu(x) \\
& \leq C_{T, r} e^{-c t^{2 \alpha-1}} C(u, f)^{2} .
\end{aligned}
$$

Let us estimate the term $\mathrm{H}_{1}$. Let $1<s<\min \{2, p\}$ and let $s^{\prime}$ be the conjugate of $s$, i.e., $\frac{1}{s}+\frac{1}{s^{\prime}}=1$. By the Hölder inequality, we have

$$
\begin{aligned}
\mathrm{H}_{1} & \leq \frac{C_{T}}{t}\|f \phi\|_{L^{s}\left(B\left(x_{0}, t^{\alpha}\right)\right)}\left\|\psi_{t} w(t, \cdot) p\left(t, x_{0}, \cdot\right)\right\|_{L^{s^{\prime}}\left(B\left(x_{0}, t^{\alpha}\right)\right)} \\
& \left.\leq \frac{C_{T}}{t}\|f\|_{L^{p}\left(B\left(x_{0}, t^{\alpha}\right)\right)} \mu\left(B\left(x_{0}, t^{\alpha}\right)\right)\right)^{\frac{1}{s}-\frac{1}{p}}\left\|\psi_{t} w(t, \cdot) p\left(t, x_{0}, \cdot\right)\right\|_{L^{s^{\prime}}\left(B\left(x_{0}, t^{\alpha}\right)\right)} .
\end{aligned}
$$

Notice that for each $t, \psi_{t} w(t, \cdot) p\left(t, x_{0}, \cdot\right) \in N_{0}^{1,2}\left(B\left(x_{0}, 2 t^{\alpha}\right)\right)$. Then by the Sobolev-Poincaré inequality (2.1), we obtain

$$
\left\|\psi_{t} w(t, \cdot) p\left(t, x_{0}, \cdot\right)\right\|_{L^{s^{\prime}\left(B\left(x_{0}, 2 t^{\alpha}\right)\right)}}
$$




$$
\left.\leq 2 t^{\alpha} \mu\left(B\left(x_{0}, 2 t^{\alpha}\right)\right)\right)^{\frac{1}{s^{\prime}}-\frac{1}{2}}\left\|D\left(\psi_{t} w(t, \cdot) p\left(t, x_{0}, \cdot\right)\right)\right\|_{L^{2}\left(B\left(x_{0}, 2 t^{\alpha}\right)\right)} \cdot
$$

Let us estimate $\left\|D\left(\psi_{t} w(t, \cdot) p\left(t, x_{0}, \cdot\right)\right)\right\|_{L^{2}\left(B\left(x_{0}, 2 t^{\alpha}\right)\right)}$. Applying the Leibniz rule, the Gaussian bounds of heat kernel (2.5), (2.6) and (3.4), we obtain

$$
\begin{aligned}
& \left\|D\left(\psi_{t} w(t, \cdot) p\left(t, x_{0}, \cdot\right)\right)\right\|_{L^{2}\left(B\left(x_{0}, 2 t^{\alpha}\right)\right)} \\
& \leq C \\
& \quad+t^{-\alpha} w(t, \cdot) p\left(t, x_{0}, \cdot\right)\left\|_{L^{2}\left(B\left(x_{0}, 2 t^{\alpha}\right)\right)}+\right\| u\left\|_{L^{\infty}\left(B\left(x_{0}, 2 r\right)\right)}\right\| t^{\alpha\left(1-\frac{Q}{2}\right)} D_{y} p\left(t, x_{0}, \cdot\right) \|_{L^{2}\left(B\left(x_{0}, 2 t^{\alpha}\right)\right)} \\
& \quad+C t^{-\alpha-\frac{Q}{4}}\left(\int_{B\left(x_{0}, 2 t^{\alpha}\right)} w^{2}(t, x) p\left(t, x_{0}, x\right) d \mu(x)\right)^{1 / 2} \\
& \quad+C t^{-\frac{Q}{4}}\left(\int_{B\left(x_{0}, 2 t^{\alpha}\right)}|D w(t, x)|^{2} p\left(t, x_{0}, x\right) d \mu(x)\right)^{1 / 2} \\
& \quad+C_{r} C(u, f) t^{\alpha\left(1-\frac{Q}{2}\right)}\left\|D_{y} p\left(t, x_{0}, \cdot\right)\right\|_{L^{2}\left(B\left(x_{0}, 2 t^{\alpha}\right)\right)}
\end{aligned}
$$

Combining the estimates (3.13)-(3.15), by using the Young inequality, we obtain

$$
\begin{aligned}
& \mathrm{H}_{1} \leq C_{T, r} t^{\alpha-1+\alpha Q\left(\frac{1}{2}-\frac{1}{p}\right)}\|f\|_{L^{p}\left(B\left(x_{0}, t^{\alpha}\right)\right)}\left\{t^{-\alpha-\frac{Q}{4}}\left(\int_{B\left(x_{0}, 2 t^{\alpha}\right)} w^{2}(t, x) p\left(t, x_{0}, x\right) d \mu(x)\right)^{1 / 2}\right. \\
& +t^{-\frac{Q}{4}}\left(\int_{B\left(x_{0}, 2 t^{\alpha}\right)}|D w(t, x)|^{2} p\left(t, x_{0}, x\right) d \mu(x)\right)^{1 / 2} \\
& \left.+C(u, f) t^{\alpha\left(1-\frac{Q}{2}\right)}\left\|D_{y} p\left(t, x_{0}, \cdot\right)\right\|_{L^{2}\left(B\left(x_{0}, 2 t^{\alpha}\right)\right)}\right\} \\
& \leq C_{T} t^{2 \alpha Q\left(\frac{1}{2}-\frac{1}{p}\right)-\frac{Q}{2}-\epsilon}\|f\|_{L^{p}\left(B\left(x_{0}, 2 r\right)\right)}^{2}+\frac{\epsilon t^{\epsilon}}{4 t^{2}} \int_{B\left(x_{0}, 2 t^{\alpha}\right)}\left|w^{2}(t, x)\right| p\left(t, x_{0}, x\right) d \mu(x) \\
& +C_{T} t^{2 \alpha-1+2 \alpha Q\left(\frac{1}{2}-\frac{1}{p}\right)-\frac{Q}{2}-\epsilon}\|f\|_{L^{p}\left(B\left(x_{0}, 2 r\right)\right)}^{2}+\frac{\epsilon t^{\epsilon}}{2 t} \int_{B\left(x_{0}, 2 t^{\alpha}\right)}|D w(t, x)|^{2} p\left(t, x_{0}, x\right) d \mu(x) \\
& +C_{T, r} t^{2 \alpha-2+2 \alpha Q\left(\frac{1}{2}-\frac{1}{p}\right)+2 \alpha\left(1-\frac{Q}{2}\right)}\|f\|_{L^{p}\left(B\left(x_{0}, 2 r\right)\right)}^{2}+C(u, f)^{2}\left\|D_{y} p\left(t, x_{0}, \cdot\right)\right\|_{L^{2}\left(B\left(x_{0}, 2 t^{\alpha}\right)\right)}^{2} \\
& =: C_{T} t^{g_{1}(\alpha, Q, p)-\epsilon}\|f\|_{L^{p}\left(B\left(x_{0}, 2 r\right)\right)}^{2}+\frac{\epsilon t^{\epsilon}}{4 t^{2}} \int_{B\left(x_{0}, 2 t^{\alpha}\right)}\left|w^{2}(t, x)\right| p\left(t, x_{0}, x\right) d \mu(x) \\
& +C_{T} t^{g^{2}(\alpha, Q, p)-\epsilon}\|f\|_{L^{p}\left(B\left(x_{0}, 2 r\right)\right)}^{2}+\frac{\epsilon t^{\epsilon}}{2 t} \int_{B\left(x_{0}, 2 t^{\alpha}\right)}|D w(t, x)|^{2} p\left(t, x_{0}, x\right) d \mu(x) \\
& +C_{T, r} t^{g_{3}(\alpha, Q, p)}\|f\|_{L^{p}\left(B\left(x_{0}, 2 r\right)\right)}^{2}+C(u, f)^{2}\left\|D_{y} p\left(t, x_{0}, \cdot\right)\right\|_{L^{2}\left(B\left(x_{0}, 2 t^{\alpha}\right)\right)}^{2} .
\end{aligned}
$$

Since $p>Q$, we have

$$
\min _{1 \leq i \leq 3}\left\{g_{i}(1 / 2, Q, p)\right\}>-1 \text {. }
$$

Since each $g_{i}$ is a continuous function of $\alpha$, there exists $\alpha \in\left(\frac{1}{3}, \frac{1}{2}\right)$ such that

$$
\min _{1 \leq i \leq 3}\left\{g_{i}(\alpha, Q, p)\right\}>-1 \text {. }
$$

Fix such an $\alpha$ and choose

$$
\epsilon \in\left(0, \frac{1}{2}+\frac{1}{2} \min _{1 \leq i \leq 3}\left\{g_{i}(\alpha, Q, p)\right\}\right)
$$


Then the above estimate reduces to

$$
\begin{aligned}
\mathrm{H}_{1} \leq & C_{T, r} t^{\epsilon-1} C(u, f)^{2}+\frac{\epsilon t^{\epsilon}}{4 t^{2}} \int_{B\left(x_{0}, 2 t^{\alpha}\right)}\left|w^{2}(t, x)\right| p\left(t, x_{0}, x\right) d \mu(x) \\
& +\frac{\epsilon t^{\epsilon}}{2 t} \int_{B\left(x_{0}, 2 t^{\alpha}\right)}|D w(t, x)|^{2} p\left(t, x_{0}, x\right) d \mu(x)+C(u, f)^{2}\left\|D_{y} p\left(t, x_{0}, \cdot\right)\right\|_{L^{2}\left(B\left(x_{0}, 2 t^{\alpha}\right)\right)}^{2} .
\end{aligned}
$$

Notice that for fixed $\beta, \gamma \in(0, \infty), t^{-\beta} e^{-t^{-\gamma}}$ is bounded on $(0, \infty)$. Applying this and the estimates of $\mathrm{H}_{1}$ and $\mathrm{H}_{2}$ to (3.10) yields

$$
\begin{aligned}
& \frac{d}{d t} J(t) \\
& \quad \geq \frac{1+\left(1-\frac{\epsilon}{2}\right) t^{\epsilon}}{t}\left(\int_{X}|D w(t, x)|^{2} p\left(t, x_{0}, x\right) d \mu(x)-\frac{1}{2 t} \int_{X} w^{2}(t, x) p\left(t, x_{0}, x\right) d \mu(x)\right) \\
& \quad-C_{T, r} C(u, f)^{2}\left[t^{\epsilon-1}-\left\|D_{y} p\left(t, x_{0}, \cdot\right)\right\|_{L^{2}\left(B\left(x_{0}, 2 t^{\alpha}\right)\right)}^{2}\right] .
\end{aligned}
$$

The estimates (3.11)-(3.12) simplify the above estimate as

$$
\frac{d}{d t} J(t) \geq-C_{T, r} C(u, f)^{2}\left[t^{\frac{1}{2}\left(1-\frac{Q}{2}\right)-1}+t^{\epsilon-1}\right]-C(u, f)^{2}\left\|D_{y} p\left(t, x_{0}, \cdot\right)\right\|_{L^{2}\left(B\left(x_{0}, 2 t^{\alpha}\right)\right)}^{2} .
$$

Integrating over $t$ on $[0, T]$ we conclude that

$$
\begin{aligned}
\int_{0}^{T} J^{\prime}(t) d t \geq & \int_{0}^{T}-C_{T, r} C(u, f)^{2}\left[t^{\frac{1}{2}\left(1-\frac{Q}{2}\right)-1}+t^{\epsilon-1}\right] d t \\
& -C(u, f)^{2} \int_{0}^{T} \int_{B\left(x, 2 t^{\alpha}\right)}\left|D_{y} p\left(t, x_{0}, y\right)\right|^{2} d \mu(x) d t \\
\geq & -C_{T, r} C(u, f)^{2}-C(u, f)^{2} \int_{0}^{T} \int_{B\left(x, 2 t^{\alpha}\right)}\left|D_{y} p\left(t, x_{0}, y\right)\right|^{2} d \mu(x) d t .
\end{aligned}
$$

Let us estimate the last term. To this end, let us recall the following inequality established in $[15,(13)]$. For every $x \in X$,

$$
\begin{aligned}
& \int_{T_{0}}^{T_{1}} \int_{B\left(x, R_{1}\right)}\left|D_{y} p(t, x, y)\right|^{2} d \mu(y) d t \\
& \quad \leq C\left[\frac{1}{\left(R_{2}-R_{1}\right)^{2}}+\frac{1}{\left(T_{2}-T_{1}\right)^{2}}\right] \int_{T_{0}}^{T_{2}} \int_{B\left(x, R_{2}\right)} p(t, x, y)^{2} d \mu(y) d t,
\end{aligned}
$$

whenever $0<R_{1}<R_{2}$ and $0 \leq T_{0}<T_{1}<T_{2}$, where $C$ is a positive constant independent of $R_{1}, R_{2}, T_{0}, T_{1}, T_{2}$ and $x$. Since $\alpha \in\left(\frac{1}{3}, \frac{1}{2}\right)$, we have that $t^{\alpha} \leq T^{1 / 3} \leq r$ and $B\left(x_{0}, 2 t^{\alpha}\right) \subset B\left(x_{0}, 2 r\right)$. By these facts and $Q \in[1,2)$, we obtain

$$
\begin{aligned}
& \int_{0}^{T} \int_{B\left(x, 2 t^{\alpha}\right)}\left|D_{y} p\left(t, x_{0}, y\right)\right|^{2} d \mu(x) d t \leq \int_{0}^{T} \int_{B(x, 2 r)}\left|D_{y} p\left(t, x_{0}, y\right)\right|^{2} d \mu(x) d t \\
& \quad \leq C\left[\frac{1}{r^{2}}+\frac{1}{T^{2}}\right] \int_{0}^{2 T} \int_{B(x, 3 r)} p(t, x, y)^{2} d \mu(y) d t
\end{aligned}
$$




$$
\leq C_{T, r} \int_{0}^{2 T} \int_{B(x, 3 r)} t^{-\frac{Q}{2}} p(t, x, y) d \mu(y) d t \leq C_{T, r} .
$$

Thus in both cases, we obtain

$$
\int_{0}^{T} \frac{d}{d t} J(t) d t \geq-C_{T, r}\left(\|u\|_{L^{2}\left(B\left(x_{0}, 4 r\right)\right)}+\|f\|_{L^{p}\left(B\left(x_{0}, 4 r\right)\right)}\right)^{2}
$$

which completes the proof of Proposition 3.2.

Proof of Proposition 3.3. By (3.9), (2.5) and (3.4), we see that

$$
\begin{aligned}
& \left|\int_{0}^{t} \int_{X} w(s, x) p\left(s, x_{0}, x\right) \phi(x) A u(x) d \mu(x) d s\right| \\
& \quad \leq C C(u, f) \int_{0}^{t} \int_{X}\left(d\left(x, x_{0}\right)^{\delta}+s^{\delta / 2}\right) s^{-\frac{Q}{2}} e^{-\frac{d\left(x, x_{0}\right)^{2}}{C_{1} s}}|\phi(x) f(x)| d \mu(x) d s \\
& \quad \leq C C(u, f) \int_{0}^{t} s^{\delta / 2} T_{l s}(|\phi f|)\left(x_{0}\right) d s,
\end{aligned}
$$

where $l=\frac{C_{1}}{2 C_{2}}$. By the fact that $T_{t}-I \rightarrow 0$ in the strong operator topology as $t \rightarrow 0$, we obtain

$$
\begin{aligned}
& \lim _{t \rightarrow 0^{+}}\left|\frac{1+t^{\epsilon}}{t} \int_{0}^{t} \int_{X} w(s, x) p\left(s, x_{0}, x\right) \phi(x) A u(x) d \mu(x) d s\right| \\
& \quad \leq C_{T} \lim _{t \rightarrow 0^{+}} \frac{1}{t} \int_{0}^{t} s^{\delta / 2} T_{l s}(|\phi f|)\left(x_{0}\right) d s=C_{T} \lim _{s \rightarrow 0^{+}} s^{\delta / 2} T_{l s}(|\phi f|)\left(x_{0}\right)=0,
\end{aligned}
$$

for almost every $x_{0} \in \Omega$.

By (3.16), we further obtain

$$
\begin{aligned}
\lim _{t \rightarrow 0^{+}} J(t) & =\lim _{t \rightarrow 0^{+}} \frac{1+t^{\epsilon}}{t} \int_{0}^{t} \int_{X}|D w(s, x)|^{2} p\left(s, x_{0}, x\right) d \mu(x) d s \\
& =\lim _{s \rightarrow 0^{+}} T_{s}\left(|D(u \phi)|^{2}\right)\left(x_{0}\right)=\left|D(u)\left(x_{0}\right)\right|^{2}
\end{aligned}
$$

for almost every $x_{0} \in \Omega$, proving the Proposition.

\section{Some applications}

In this section, we discuss some sufficient conditions for (1.1). As pointed out in the introduction, the logarithmic inequality (1.2) guarantees $(1.1)$; see $[2,9,10]$ for more about the logarithmic inequality. Moreover, there is a result about curvature that guarantees (1.1). Let us first recall some notions; see, for instance, $[1,2,15]$.

For all $u, v, u v \in D(A)$, define the "square of the length of the gradient" pointwise by

$$
\Gamma(u, v)(x)=\frac{1}{2}[A(u v)(x)-u(x) A v(x)-v(x) A u(x)] .
$$


Further, assume that there exists a dense subspace $\mathscr{S} \subset N^{1,2}(X)$ such that for all $u, v \in \mathscr{S}$, we can define

$$
\Gamma_{2}(u, v)(x)=\frac{1}{2}[A(\Gamma(u, v))(x)-\Gamma(u, A v)(x)-\Gamma(v, A u)(x)] .
$$

The diffusion semigroup is said to have curvature greater or equal to some $\kappa \in \mathbb{R}$, if for all $u \in \mathscr{S}$ and $x \in X$,

$$
\Gamma_{2}(u, u) \geq \kappa \Gamma(u, u) .
$$

The following result is part of [1, Proposition 2.1].

Proposition 4.1. Assume that the subspace $\mathscr{S}$ is as above, and that the diffusion semigroup has curvature greater or equal to some $\kappa \in \mathbb{R}$. Then, for every $u \in N^{1,2}(X)$, each $t>0$ and for almost every $x_{0} \in X$, it holds

$$
\int_{X}\left(u(x)-T_{t} u(x)\right)^{2} p\left(t, x_{0}, x\right) d \mu(x) \leq \frac{1-e^{-2 \kappa t}}{\kappa} \int_{X}|D u(x)|^{2} p\left(t, x_{0}, x\right) d \mu(x),
$$

when $\kappa=0, \frac{1-e^{-2 k t}}{\kappa}$ is replaced by $2 t$. Moreover, if inequality (4.2) holds true for every $u \in N^{1,2}(X)$ and almost every $x_{0} \in X$, then (4.1) holds true for all functions in some dense subclass $\mathscr{S} \subset$ $N^{1,2}(X)$ as well.

Since (4.2) implies (1.1), by Proposition 4.1, we see that (1.1) holds when the curvature of the diffusion semigroup is bounded from below. It is well known that Riemannian manifolds with Ricci curvature bounded from below satisfies (4.1), where the generator $A$ is the Laplace-Beltrami operator; see, for example, [1,7].

Another example given in [15] is the Euclidean spaces with smooth Ahlfors regular weights. Let $w \in C^{2}\left(\mathbb{R}^{n}\right)$ be an Ahlfors regular weight. It was shown that if $\frac{1}{w^{2}}\left(|\nabla w|^{2}-w \Delta w\right) \geq \kappa$, then for all $u \in C_{0}^{\infty}\left(\mathbb{R}^{n}\right), \Gamma_{2}(u, u) \geq \kappa \Gamma(u, u)$. Here, we want to give another explanation which shows that $\Gamma_{2}(u, u) \geq 0$ whenever $w$ is a positive $C^{2}\left(\mathbb{R}^{n}\right)$ function. For every $u \in C_{0}^{\infty}\left(\mathbb{R}^{n}\right)$, we have $\Gamma(u, u)=|\nabla u|^{2}$, and

$$
\begin{aligned}
\int_{\mathbb{R}^{n}} & \Gamma_{2}(u, u)(x) w(x) d x \\
= & \int_{\mathbb{R}^{n}} \frac{1}{2}\left[A\left(|\nabla u|^{2}\right)(x)-2 \nabla u(x) \cdot \nabla(A u)(x)\right] w(x) d x \\
= & -\int_{\mathbb{R}^{n}} \nabla u(x) \cdot \nabla(\Delta u)(x) w(x) d x-\int_{\mathbb{R}^{n}} \nabla u(x) \cdot \nabla\left(\frac{\nabla u \cdot \nabla w}{w}\right)(x) w(x) d x \\
= & \int_{\mathbb{R}^{n}}(\Delta u(x))^{2} w(x) d x+\int_{\mathbb{R}^{n}} \Delta u(x) \nabla u(x) \cdot \nabla w(x) d x \\
& +\int_{\mathbb{R}^{n}} \Delta u(x) \nabla u(x) \cdot \nabla w(x) d x+\int_{\mathbb{R}^{n}} \frac{|\nabla u(x) \cdot \nabla w(x)|^{2}}{w(x)} d x \\
= & \int_{\mathbb{R}^{n}}\left(\Delta u(x) \sqrt{w(x)}+\frac{\nabla u(x) \cdot \nabla w(x)}{\sqrt{w(x)}}\right)^{2} d x \geq 0 .
\end{aligned}
$$

Thus (4.1) always holds with $\kappa=0$, whenever $w \in C^{2}\left(\mathbb{R}^{n}\right)$ is positive. Notice here, the condition that $w \in C^{2}\left(\mathbb{R}^{n}\right)$ is positive implies that $w$ is a locally Ahlfors-regular weight. 


\section{Acknowledgment}

The author is grateful to his supervisor Professor Pekka Koskela for posing the problem and many kind suggestions. He also wishes to express deeply thanks to Kai Rajala, Guo Zhang and Yuan Zhou for many helpful discussions. Last but not least, the author would also like to thank the referee for his many valuable remarks which made this article more readable.

\section{References}

[1] D. Bakry, On Sobolev and Logarithmic Inequalities for Markov Semigroups, New Trends in Stochastic Analysis (Charingworth, 1994), World Scientific Publishing, River Edge, NJ, 1997, pp. 43-75.

[2] D. Bakry, M. Emery, Diffusions hypercontractives, Seminaire de probabilities, Vol. XIX, 1983/84, pp. 177-206.

[3] M. Biroli, U. Mosco, Sobolev inequalities for Dirichlet forms on homogeneous spaces. Boundary value problems for partial differential equations and applications, RMA Res. Notes Appl. Math., 29, Masson, Paris, (1993) 305-311.

[4] M. Biroli, U. Mosco, A Saint-Venant type principle for Dirichlet forms on discontinuous media, Ann. Mat. Pura Appl. 169 (1995) 125-181.

[5] L.A. Caffarelli, C.E. Kenig, Gradient estimates for variable coefficient parabolic equations and singular perturbation problems, Amer. J. Math. 120 (1998) 391-439.

[6] J. Cheeger, Differentiability of Lipschitz functions on metric measure spaces, Geom. Funct. Anal. 9 (1999) 428-517.

[7] S.Y. Cheng, S.T. Yau, Differential equations on Riemannian manifolds and their geometric applications, Comm. Pure Appl. Math. 28 (3) (1975) 333-354.

[8] M. Fukushima, Y. Oshima, M. Takeda, Dirichlet Forms and Symmetric Markov Processes, in: de Gruyter Studies in Mathematics, Vol. 19, Walter de Gruyter \& Co., Berlin, 1994.

[9] L. Gross, Logarithmic Sobolev inequalities, Amer. J. Math. 97 (1975) 1061-1083.

[10] L. Gross, Hypercontractivity over complex manifolds, Acta Math. 182 (1999) 159-206.

[11] P. Hajłasz, P. Koskela, Sobolev meets Poincaré, C. R. Acad. Sci. Paris Sér. I Math. 320 (10) (1995) 1211-1215.

[12] P. Hajłasz, P. Koskela, Sobolev met Poincaré, Mem. Amer. Math. Soc. 145 (688) (2000).

[13] J. Heinonen, P. Koskela, Quasiconformal maps in metric spaces with controlled geometry, Acta Math. 181 (1998) 1-61.

[14] T. Kilpeläinen, J. Kinnunen, O. Martio, Sobolev spaces with zero boundary values on metric spaces, Potential Anal. 12 (3) (2000) 233-247.

[15] P. Koskela, K. Rajala, N. Shanmugalingam, Lipschitz continuity of Cheeger-harmonic functions in metric measure spaces, J. Funct. Anal. 202 (2003) 147-173.

[16] L. Saloff-Coste, A note on Poincaré, Sobolev, and Harnack inequalities, Internat. Math. Res. Notices (2) (1992) 27-38.

[17] S. Semmes, in: M. Gromov (Ed.), Metric Structures for Riemannian and Non-Riemannian Spaces, Appendix, Progress in Mathematics, Vol. 152, Birkhäuser Boston, Inc., Boston, MA, 1999. 
[18] N. Shanmugalingam, Newtonian spaces: an extension of Sobolev spaces to metric measure spaces, Rev. Mat. Iberoamericana 16 (2000) 243-279.

[19] K.T. Sturm, Analysis on local Dirichlet spaces. I. Recurrence, conservativeness and $L^{p}$ Liouville properties, J. Reine Angew. Math. 456 (1994) 173-196.

[20] K.T. Sturm, Analysis on local Dirichlet spaces. III. The parabolic Harnack inequality, J. Math. Pures Appl. (9) 75 (3) (1996) 273-297.

\section{Renjin Jiang}

Department of Mathematics and Statistics

University of Jyväskylä

P.O. Box $35(\mathrm{MaD})$

FI-40014

Finland

E-mail address: renjin.r.jiang@jyu.fi 